\title{
Rede virtual: ferramenta para analisar o conhecimento dos voluntários do MORHAN sobre
} hanseníase

\author{
Virtual network: to analyze the knowledge about leprosy of MORHAN volunteers
}

\section{Red virtual: herramienta para analizar el conocimiento de los voluntarios del MORHAN sobre lepra}

Recebido: 13/06/2015

Aprovado: 31/03/2016

Publicado: 01/05/2016

\section{Susilene Maria Tonelli Nardi ${ }^{1}$ Heloisa da Silveira Paro Pedro² Luisa Arantes Loureiro ${ }^{3}$ Lucia Helena Soares Camargo Marciano ${ }^{4}$ Artur Custódio Correa de Sousa ${ }^{5}$ Vânia Del'Arco Paschoal ${ }^{6}$}

O objetivo do estudo foi revelar o conhecimento sobre hanseníase dos voluntários participantes da Rede Virtual de Mobilização (REMOB) do Movimento de Reintegração das pessoas Atingidas pela Hanseníase (MORHAN) em relação a aspectos gerais, diagnóstico, transmissão e tratamento. Os voluntários da REMOB foram convidados a responder um formulário online contendo 35 afirmativas sobre hanseníase e perguntas específicas sobre o voluntariado. Participaram do estudo 105 voluntários, representando 20 estados brasileiros, a média de idade foi de 38,9 (dp=14,14), 72,4\% eram atuantes na área da saúde, 73,3\% tinham nível superior e/ou pós-graduação, para 63,8\% as atividades voluntárias não iniciaram por conhecer alguém com a doença. 0 percentual médio de acertos sobre diagnóstico foi de $84,2 \%$, aspectos gerais $83,2 \%$, transmissão $71,6 \%$ e tratamento $61,9 \%$. 0 conhecimento sobre hanseníase foi maior nas questões referentes ao diagnóstico e menor sobre tratamento sendo imprescindível investimento em capacitação permanente.

Descritores: Hanseníase; Educação em saúde; Voluntários; Rede social; Conhecimento.

The objective of this study was to investigate knowledge about leprosy of volunteers participating in the Virtual Mobilization Network (REMOB) for the Reintegration Movement of People Affected by Leprosy (MORHAN) in relation to general aspects, diagnosis, transmission and treatment of the disease. REMOB volunteers were asked to answer an online questionnaire containing 35 questions about leprosy and specific questions about volunteering. The study included 105 volunteers in 20 Brazilian states with a mean age of 38.9 ( $\mathrm{sd}=14.14$ ) years. A total of $72.4 \%$ were healthcare professionals, $73.3 \%$ had college diplomas or were postgraduates, and for $63.8 \%$ volunteering did not begin because they knew someone with the disease. Respondents correctly answered, on average, $84.2 \%$ of the questions about diagnosis, $83.2 \%$ about general aspects, $71.6 \%$ about transmission and $61.9 \%$ on treatment. The knowledge of leprosy was better in respect to the diagnosis and worse in relation to treatment and should be the target for investment in ongoing training.

Descriptors: Leprosy; Health education; Volunteers; Social networking; Knowledge

El objetivo de este estudio es revelar el conocimiento sobre lepra de los voluntarios de la Rede Virtual de Movilización (REMOB) del Movimiento de Reintegración de Personas Afectadas por la Lepra (MORHAN) en: aspectos generales, diagnóstico, transmisión y tratamiento. Los voluntarios de REMOB respondieron un formulario online conteniendo 35 afirmaciones sobre Lepra y preguntas específicas acerca del voluntariado. Participaron en el estudio 105 voluntarios, representando 20 estados brasileños, la edad promedio fue 38,9 ( $\mathrm{dp}=14,14) ; 72,4 \%$ estaban activos en área de salud; 73,3\% tenían nivel superior y/o posgrado; el 63,8\% no iniciaron las actividades voluntarias por conocer a alguien con la enfermedad. El porciento promedio de aciertos en diagnóstico fue 84,2\%, en aspectos generales $83,2 \%$, en transmisión $71,6 \%$ y en tratamiento $61,9 \%$. Se concluyó que el conocimiento sobre lepra fue mayor en cuestiones referentes al diagnóstico y menor referente al tratamiento, deben ser indispensable para la capacitación permanente.

Descriptores: Lepra; Educación en salud; Voluntarios; Red social; Conocimiento.

\footnotetext{
${ }^{1}$ Terapeuta Ocupacional. Mestre e Doutora em Ciências da Saúde. Voluntária MORHAN- Movimento da Reintegração das Pessoas Atingidas pela Hanseníase. Pesquisadora Científica VI do Instituto Adolfo Lutz (IAL) - São José do Rio Preto/SP. susilenenardi@gmail.com. Brasil.

${ }^{2}$ Bióloga. Mestre em Microbiologia. Doutora em Genética. Pesquisadora Científica do IAL- São José do Rio Preto/SP. Voluntária da MORHAN. hsppedro@ial.sp.gov.br. Brasil.

${ }^{3}$ Terapeuta Ocupacional. Mestre em Saúde Coletiva. Professora Assistente da Universidade Federal do Triângulo Mineiro. luisa.loureiro@gmail.com. Brasil.

${ }^{4}$ Terapeuta Ocupacional. Mestre em Reabilitação. Doutora em Ciências da Saúde. Pesquisadora Científica do Instituto Lauro de Souza Lima, Bauru/SP. Voluntária da MORHAN. Imarciano@ilsl.br. Brasil.

${ }^{5}$ Administrador de Empresas. Mestre em Empreendedorismo Social. Responsável e criador da MORHAN. Administrador de Empresas da MassChallenge - Aceleradora de Empresas - Boston/MA - USA. arturcsousa@gmail.com. Brasil.

${ }^{6}$ Enfermeira. Mestre e Doutor em Ciências da Saúde. Professora Adjunta da Faculdade de Medicina de São José do Rio Preto. Coordenadora Suplente do Curso de Graduação em Enfermagem da FAMERP. Voluntária da MORHAN. vaniadelarco@gmail.com. Brasil.
} 


\section{INTRODUÇÃO}

$\mathrm{A}$ hanseníase causa preocupação a quem a contrai, aos profissionais de saúde, à população e aos órgãos governamentais e não-governamentais, tanto pelas implicações físicas e sociais, quanto pela quantidade de pessoas diagnosticadas anualmente no mundo ${ }^{1}$.

Os planos de eliminação da doença elaborados pela Organização Mundial de Saúde (OMS) e posteriormente pelo Ministério da Saúde apresentam como componente mais importante assegurar que as atividades de controle da hanseníase tivessem ao alcance de toda população, bem como o acesso ao diagnóstico, tratamento com poliquimioterapia (PQT), aconselhamento aos pacientes e familiares, educação comunitária, prevenção e reabilitação de deficiências/incapacidades e encaminhamento das complicações ${ }^{2,3}$.

Destaca-se entre os mecanismos de sustentação das atividades de controle da hanseníase "o desenvolvimento da capacidade $e$ competência dos profissionais de atenção à saúde por meio da capacitação e atualização em serviços"2. Dessa forma, torna-se fundamental investir em técnicas de educação em saúde com o objetivo de informar, prevenir ou minimizar as sequelas decorrentes dessa condição clínica com objetivo de interromper a cadeia epidemiológica da doença ${ }^{4,5}$, haja vista que a educação em saúde é compreendida como processo de transformação que desenvolve a consciência crítica das pessoas a respeito de seus problemas de saúde e estimula a busca de soluções coletivas para resolvê-los"6.

Na hanseníase, a educação em saúde possibilita o desenvolvimento de ações educativas voltadas para conhecimento da doença pela sociedade e consequente melhoria da saúde do paciente e de seus familiares ${ }^{7}$. Em uma abordagem prática a educação em saúde conscientiza e sensibiliza o indivíduo a participar da vida comunitária de uma maneira construtiva, preparando-o não apenas para a prevenção de doenças, mas para obter ou manter uma vida mais saudável ${ }^{8}$.
O Movimento de Reintegração das Pessoas Atingidas Pela Hanseníase (MORHAN) é uma entidade sem fins lucrativos, criado em 1981 e tem como missão possibilitar que a hanseníase seja compreendida na sociedade como uma doença normal, com tratamento e cura, eliminando assim o preconceito em torno da doença.

A força de ação do MORHAN se pauta no voluntariado, e tem suas atividades voltadas para a eliminação da hanseníase, buscando a construção de políticas públicas eficazes por meio de conscientização da população sobre o problema e foco na garantia de ações de prevenção, tratamento, diagnóstico e reabilitação para as pessoas atingidas pela doença9.

Com vistas a cadastrar os voluntários já participantes do MORHAN e fortalecer a militância, mobilização e ampliar o número de voluntários, em 2010 foi criada a Rede Virtual de Mobilização, o REMOB, cujo cadastro se dá pelo link http://www.morhan.org.br/participe que facilitou a pulverização das ações do MORHAN nas redes sociais. A adesão no REMOB de todos os voluntários já participantes do MORHAN não ocorreu devido às dificuldades individuais de acesso a ferramentas tecnológicas.

Nesse contexto, é importante que os voluntários do REMOB tenham conhecimentos básicos sobre a doença como parte de um processo de educação em saúde, para um esforço concentrado nas três instâncias do SUS na busca da efetividade máxima das ações de controle da hanseníase. Estudos que avaliem esse conhecimento são necessários haja vista a amplitude de ação dos voluntários em um movimento social, que frequentemente mantém contato direto com pessoas que tem ou tiveram hanseníase e que por vezes se sentem despreparados para compreender e elucidar as dúvidas que surgem durante esse contato e ou durante o processo de reivindicação de direitos.

Para tanto o objetivo desse estudo foi revelar quantitativamente o conhecimento dos voluntários participantes da Rede Virtual de 
Mobilização do MORHAN, sobre hanseníase em relação a aspectos gerais, diagnóstico, tratamento e transmissão da doença.

\section{MÉTODO}

A população foi constituída por voluntários do MORHAN, que fazem parte e estão cadastrados na Rede de Mobilização do MORHAN (REMOB). A REMOB é uma rede virtual de voluntários do MORHAN, cujos integrantes devem estar necessariamente cadastrados online, podendo atuar ou não em outras ações do Movimento, que não exclusivamente de forma virtual.

0 critério de inclusão foi apenas a aceitação do voluntário cadastrado na REMOB em participar desse estudo, respondida no próprio protocolo do pré-teste sua aceitação e concordância após leitura do Termo de Consentimento Livre e Esclarecido.

0 instrumento de coleta de dados utilizado nesse estudo é composto de 35 afirmativas e foi preenchido por meio do link disponibilizado ${ }^{10}$ para os voluntários que deveriam inserir o no do R.G, iniciais do nome, data de nascimento, gênero, grau de escolaridade, profissão, ocupação atual (cargo); área de atuação, município de residência, estado e responder perguntas específicas sobre o seu voluntariado.

Esse instrumento de coleta (pré-teste) foi elaborado inicialmente pelo Núcleo de Educação em Saúde do Centro de Vigilância Epidemiológica em 2001, modificado em dezembro de 2005 por Nardi SMT, Marciano LHSC, Prado RBR e Quaggio CMP11 e em outubro de 2011 para adequação da versão online foi novamente modificado por Nardi SMT; Pedro HSP; Sousa ACC; Loureiro LA; Paschoal VDA.

As modificações realizadas no pré-teste pelos pesquisadores em $2005^{11}$ e posteriormente em 2011 foram baseadas no Álbum Seriado ${ }^{12}$ da hanseníase, financiado pela Fundação Paulista Contra Hanseníase que foi criado com objetivo de facilitar atividades educativas ou grupais sobre a doença. 0 álbum seriado é grande aliado na educação em saúde direcionada à hanseníase, pois contém os conceitos básicos e mínimos sobre definição, transmissão, sinais, sintomas, formas clínicas, tratamento medicamentoso, autocuidados, direitos e deveres do paciente.

Posteriormente a aplicação do pré-teste os participantes eram convidados e direcionados por meio de um link para a página inicial do MORHAN para leitura e conhecimento do álbum seriado ${ }^{12}$ que traz em seu conteúdo a resposta para todas as questões elaboradas no pré-teste.

Realizou-se um projeto piloto com 30 voluntários da REMOB (MORHAN), que não entraram na população deste estudo. Os mesmos responderam o formulário disponível no site, com propósito de possível ajuste da metodologia aplicada e adequação da logística utilizada no projeto com os demais voluntários.

A coleta de dados foi realizada no período de junho de 2012 a março de 2013, na qual os voluntários foram convidados a responder o questionário eletrônico disponível em:

http://www.formfacil.com/susitn/preteste ${ }^{10}$.

Após os participantes enviarem o préteste respondido, uma planilha em Excel@ foi gerada para posterior analise das variáveis.

Utilizou-se programa estatístico para o levantamento de frequências, médias e cruzamento dos dados, considerando as variáveis referentes à caracterização dos participantes e respectivas respostas do préteste de cada um deles. Foram considerados os resultados que apresentarem $5 \%$ de significância e poder de $80 \%$.

As questões contidas no formulário foram subdividas em quatro categorias: Aspectos Gerais (10 questões), Diagnóstico (10 afirmativas), Tratamento (08 afirmativas) e Transmissão (07 questões). Os escores foram categorizados em nível de conhecimento conforme proposto por Marciano et $\mathrm{al}^{11}$ considerando superior à média (>50\%) e inferior à média $(\leq 50 \%)$ considerando acertos e erros registrados no pré-teste.

Para a apresentação dos resultados, as palavras "afirmativa correta" e "afirmativa 
incorreta" foram inseridas em seguida às frases propostas no pré-teste.

0 projeto foi aprovado pelo Comitê de Ética em Pesquisa do Instituto Lauro de Souza Lima (CEP/ILSL) protocolo 231/11 em 28 de fevereiro de 2012.

\section{RESULTADOS}

De um total de 127 inscritos no REMOB/MORHAN no período desse estudo, $105(82,7 \%)$ voluntários responderam ao préteste online, com representatividade de 20 estados do Brasil, sendo Minas Gerais (24,8\%) e São Paulo (20,0\%) os mais frequentes.

Os participantes representavam vários segmentos de atuação, com destaque para a Saúde $(n=76 / 72,4 \%)$ seguido de educação $(n=9 / 8,6 \%)$.

Houve representação de várias profissões, entre elas as que mais apareceram foram estudante $(26,7 \%)$, enfermeiro $(10,5 \%)$, assistente social (4,8\%), farmacêutico bioquímico $(4,8 \%)$, professor $\quad(4,7 \%)$, terapeuta ocupacional (3,8\%), psicólogo $(2,9 \%)$ e outras $(41,8 \%)$.

Em relação à escolaridade dos participantes, 3,8\% (4) tinham ensino fundamental, $22,9 \%$ (24) tinham o segundo grau completo, $39 \%$ (41) o ensino superior completo, 34,3\% (36) pós-graduação. A idade média dos voluntários foi de 38,9 (dp=14,14) com variação de 17 a 71 anos.

Dos voluntários, 53,3\% (56) respondeu que conhecia amigo ou familiar que tem ou teve hanseníase, mas apenas 36,2\% (38) iniciaram as atividades de voluntariado pelo fato de ter alguém conhecido com a doença e 80\% (84) tinha algum conhecimento sobre a doença antes de iniciar seu voluntariado.

Salienta-se que 37,2\% dos voluntários desconheciam que a única fonte de transmissão conhecida atualmente é o homem (Tabela 1).

Tabela 1. Distribuição de frequência de acertos, erros e "não sabe" das questões relativas a aspectos gerais sobre hanseníase. São Paulo, Março de 2013.

AFIRMATIVAS

- Existe outra fonte de infecção de hanseníase além do homem. "afirmativa incorreta"

- O paciente com hanseníase deve evitar o contato físico (relações sexuais, beijo, abraço, outros) e ambientes coletivos (banheiro, ônibus, piscina) e manter seus objetos de uso pessoal separado. "afirmativa incorreta"

- Entre as pessoas que adoecem, alguns desenvolvem poucas manchas com poucos bacilos e outras apresentam formas mais graves com muitos bacilos. "afirmativa correta"

- Nem todas as formas de Hanseníase são contagiosas. As contagiosas deixam de ser quando o tratamento é iniciado. "afirmativa correta"

- A Hanseníase é uma doença que ataca a pele e nervos e pode causar deformidades nos olhos, no nariz, nas mãos e nos pés. "afirmativa correta"

- A cura da Hanseníase ainda não foi alcançada. "afirmativa incorreta"

- A Hanseníase é hereditária. "afirmativa incorreta"

- O paciente de hanseníase deve ser tratado de forma diferenciada na rede de saúde pública. "afirmativa incorreta"

- O paciente de Hanseníase deve continuar sua vida normal junto à família, amigos e trabalho. "afirmativa correta"

- Somente o médico poderá suspeitar de Hanseníase e realizar os encaminhamentos necessários. "afirmativa incorreta"

\begin{tabular}{|c|c|c|c|c|c|}
\hline \multicolumn{2}{|c|}{ ACERTOS } & \multicolumn{2}{|c|}{ ERROS } & \multicolumn{2}{|c|}{ NÃO SABE } \\
\hline$\%$ & $\mathrm{~N}$ & $\%$ & $\mathrm{n}$ & $\%$ & $\mathrm{n}$ \\
\hline 62,9 & 66 & 14,3 & 15 & 22,9 & 24 \\
\hline 81,0 & 85 & 15,2 & 16 & 3,8 & 4 \\
\hline 79,1 & 83 & 5,7 & 6 & 15,3 & 16 \\
\hline 81,0 & 85 & 10,5 & 11 & 8,6 & 9 \\
\hline 90,5 & 95 & 4,8 & 5 & 4,8 & 5 \\
\hline 85,7 & 90 & 10,5 & 11 & 3,8 & 4 \\
\hline 89,5 & 94 & 4,8 & 5 & 5,7 & 6 \\
\hline 83,8 & 88 & 8,6 & 9 & 7,6 & 8 \\
\hline 97,1 & 102 & - & - & 2,9 & 3 \\
\hline 80,0 & 84 & 18,1 & 19 & 1,9 & 2 \\
\hline
\end{tabular}


De acordo com a Tabela 2, dentre os sinais e sintomas o conhecimento mais evidente dos voluntários é mancha que não incomoda e que é dormente. Outras características das manchas que podem alertar para o diagnóstico parecem ser conceitos menos conhecidos, como lesão das fibras autonômicas que ocasiona queda de pêlo, falta de transpiração e ressecamento da pele, fazendo com que a poeira não fixe na pele.

Já, a transmissão pelas formas da doença e pelas mulheres grávidas e/ou que amamentam são desconhecidas pela maioria dos voluntários, conforme apresentado na Tabela 3. Muitas questões sobre o tratamento são dúbias, em especial os efeitos colaterais dos medicamentos e a forma de administração medicamentosa (Tabela 4).

Tabela 2. Distribuição de frequência de acertos, erros e "não sabe" das questões relativas a diagnóstico sobre hanseníase. São Paulo, Março de 2013.

\begin{tabular}{|c|c|c|c|c|c|c|}
\hline \multirow[t]{2}{*}{ AFIRMATIVAS } & \multicolumn{2}{|c|}{ ACERTOS } & \multicolumn{2}{|c|}{ ERROS } & \multicolumn{2}{|c|}{ NÃO SABE } \\
\hline & $\%$ & $\mathrm{n}$ & $\%$ & $\mathrm{n}$ & $\%$ & $\mathrm{n}$ \\
\hline $\begin{array}{l}\text { - Manchas na pele que não doem, não incomodam. "afirmativa } \\
\text { correta" }\end{array}$ & 93,3 & 98 & 5,7 & 6 & 1,0 & 1 \\
\hline - Manchas que coçam. "afirmativa incorreta" & 75,2 & 79 & 13,3 & 14 & 11,4 & 12 \\
\hline - Manchas com queda de pêlo. "afirmativa correta" & 70,5 & 74 & 10,5 & 11 & 19,1 & 20 \\
\hline $\begin{array}{l}\text { - Áreas/ manchas com dormência ou formigamento. "afirmativa } \\
\text { correta" }\end{array}$ & 91,4 & 96 & 4,8 & 5 & 3,8 & 4 \\
\hline - Manchas que pegam pó. "afirmativa incorreta" & 55,2 & 58 & 5,7 & 6 & 39,1 & 41 \\
\hline - Queimar-se ou cortar-se sem sentir. "afirmativa correta" & 93,3 & 98 & 1,9 & 2 & 4,8 & 5 \\
\hline $\begin{array}{l}\text { - Manchas esbranquiçadas ou avermelhadas. "afirmativa correta" } \\
\text { - Na hanseníase a lesão dos nervos periféricos pode ocasionar dor, }\end{array}$ & 91,4 & 96 & 5,7 & 6 & 2,9 & 3 \\
\hline $\begin{array}{l}\text { fraqueza diminuição da sensibilidade. "afirmativa correta" } \\
\text { - Diante de um caso com suspeita de hanseníase, o médico, deverá } \\
\text { minimamente examinar pele, nervos, sensibilidade e solicitar exames }\end{array}$ & 95,2 & 100 & 1,9 & 2 & 2,9 & 3 \\
\hline $\begin{array}{l}\text { laboratoriais, se necessário. "afirmativa correta" } \\
\text { - A baciloscopia (coleta da linfa) e a biopsia (retirada de um } \\
\text { pedacinho da pele da mancha) são exames de laboratório para } \\
\text { verificar a presença de bacilos no corpo. "afirmativa correta" }\end{array}$ & 90,5 & 95 & 4,8 & 5 & 4,8 & 12 \\
\hline
\end{tabular}

Tabela 3. Distribuição de frequência de acertos, erros e "não sabe" das questões relativas a transmissão sobre hanseníase. São Paulo, Março de 2013.

\begin{tabular}{|c|c|c|c|c|c|c|}
\hline \multirow{2}{*}{ AFIRMATIVAS } & \multicolumn{2}{|c|}{ ACERTOS } & \multicolumn{2}{|c|}{ ERROS } & \multicolumn{2}{|c|}{ NÃO SABE } \\
\hline & $\%$ & $\mathrm{n}$ & $\%$ & $\mathrm{n}$ & $\%$ & $\mathrm{n}$ \\
\hline $\begin{array}{l}\text { - Os bacilos de um doente sem tratamento passam para as pessoas } \\
\text { sadias, principalmente através de contatos diretos e frequentes. } \\
\text { "afirmativa correta" }\end{array}$ & 86,7 & 91 & 8,6 & 9 & 4,8 & 5 \\
\hline $\begin{array}{l}\text { - A hanseníase é transmitida principalmente por meio das vias } \\
\text { respiratórias. "afirmativa correta" } \\
\text { - A maioria das pessoas que entra em contato com o bacilo de }\end{array}$ & 85,7 & 90 & 10,5 & 11 & 1,7 & 4 \\
\hline $\begin{array}{l}\text { Hansen adoece. "afirmativa incorreta" } \\
\text { - As pessoas que convivem com pacientes que tem ou tiveram } \\
\text { hanseníase precisam ser examinadas e acompanhadas pela equipe } \\
\text { de saúde, sendo esse um dos principais fatores que contribuem para }\end{array}$ & 78,1 & 82 & 8,6 & 9 & 13,3 & 14 \\
\hline $\begin{array}{l}\text { a "quebra" da cadeia de transmissão. "afirmativa correta" } \\
\text { - A mulher pode passar hanseníase para seu filho na gravidez e ou }\end{array}$ & 85,7 & 90 & 4,8 & 5 & 9,5 & 10 \\
\hline $\begin{array}{l}\text { durante a amamentação. "afirmativaincorreta" } \\
\text { - A forma indeterminada da doença não é contagiosa, sua cura é mais } \\
\text { rápida e fácil, se não tratada, pode evoluir para formas mais graves. }\end{array}$ & 62,9 & 66 & 11,4 & 12 & 25,7 & 27 \\
\hline $\begin{array}{l}\text { "afirmativa correta" } \\
\text { - A forma tuberculóide é contagiosa, ou seja, passa de uma pessoa }\end{array}$ & 69,5 & 73 & 9,5 & 10 & 21,0 & 22 \\
\hline $\begin{array}{l}\text { para outra. "afirmativa incorreta" } \\
\text { As formas dimorfa e virchoviana quando não tratadas são }\end{array}$ & 38,1 & 40 & 37,1 & 39 & 24,8 & 26 \\
\hline contagiosas. "afirmativa correta" & 65,7 & 69 & 5,7 & 6 & 28,6 & 30 \\
\hline
\end{tabular}


Tabela 4. Distribuição de frequências de acertos, erros e "não sabe" das questões relativas a tratamento sobre hanseníase. São Paulo, Março de 2013.

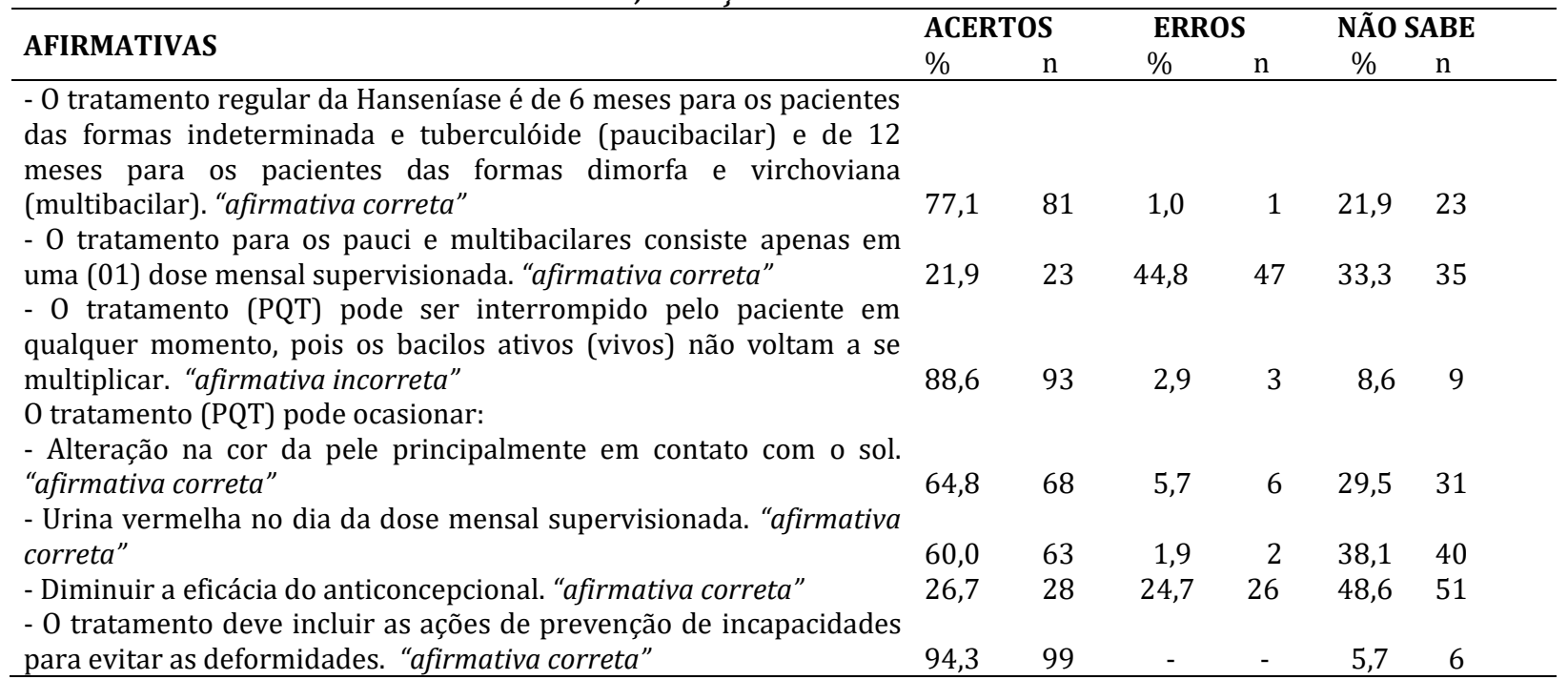

Tabela 5. Média percentual de acertos no pré teste, considerando aspectos gerais, diagnóstico, transmissão e tratamento. São Paulo, Março de 2013.

\begin{tabular}{lcccc} 
& \multicolumn{2}{c}{ PRÉ TESTE } \\
& Média* & Desvio Padrão & Mínimo & Máximo \\
\hline Aspectos Gerais & 83,1 & 17,1 & 62,9 & 97,1 \\
Diagnóstico & 84,2 & 20,0 & 55,2 & 95,2 \\
Transmissão & 71,6 & 24,3 & 38,1 & 86,7 \\
Tratamento & 61,9 & 36,2 & 21,9 & 94,3 \\
\hline *Percentual & & & &
\end{tabular}

*Percentual

DISCUSSÃO

Para ultrapassar o limite da informação e adquirir conhecimento o indivíduo precisa raciocinar e ser consciente do mundo exterior considerando sua experiência e aprendizagem prévias $^{13}$.

Os voluntários do REMOB são pessoas que se inscreveram voluntariamente no site do MORHAN e que representam um universo profissional diversificado, que trazem consigo uma bagagem de conhecimento bastante vasta.

Os estados de Minas Gerais e São Paulo tiveram uma representatividade maior no universo de pessoas desse estudo, em função do intenso trabalho realizado em algumas cidades desses estados por voluntários do MORHAN em busca de adesões no REMOB.

A hanseníase é uma doença com interfaces sociais, físicas, psicológicas e, que carece de investigações mais ampliadas em determinados campos.

As informações sobre a doença são variadas, dispersas e por vezes específicas sobremaneira para os que não atuam na área da saúde. Nessa consideração, esse trabalho buscou compreender se as pessoas que se propõem a lutar por uma causa conhecem a dimensão do assunto que estão envolvidas.

0 protocolo de investigação traz afirmativas sobre as descobertas científicas traduzidas em uma linguagem simples, familiar e mais vinculadas ao dia-a-dia e teve como proposta secundária, além de investigar os conhecimentos dos participantes, incitar a formação de uma visão ampliada e crítica sobre o assunto em questão, de forma a exercerem ativamente a cidadania e sua estratégia de ação dentro do movimento ${ }^{14}$. 
No estudo optou-se por utilizar a tela digital para a investigação, haja vista que a maioria dos participantes aderiu ao voluntariado inteiramente de forma online e isso fez com que $82,7 \%$ do total de voluntários do REMOB participassem da pesquisa. Com a tela digital gerações de utilizadores tomam uma atitude mais autoral e colaborativa diante dos conteúdos que lhes são expostos ${ }^{15}$.

Quase a metade dos participantes dessa pesquisa conhecia alguém, amigo ou familiar, que havia sido diagnosticado com a doença e iniciaram seu voluntariado por essa razão. Sabe-se que vivenciar a doença na imagem de uma pessoa próxima que se tem afetividade, sensibiliza e encoraja para a ação ${ }^{16}$.

Os resultados demonstram um conhecimento superior à média ( $>50$ ) em todas as categorias a que foram subdivididas as afirmativas do instrumento de investigação.

No grande tema sobre Aspectos Gerais, apesar de a média de acertos ter sido alta $(83,2 \%)$, algumas questões parecem causar duvidas nos participantes, como a de ter outra fonte de infecção além do homem. Embora ainda não comprovado que a doença seja uma zoonose ${ }^{17-19}$, a bactéria tem a capacidade de infectar e se multiplicar em alguns animais, sendo esses considerados como seus reservatórios naturais ${ }^{20}$.

Interessante também foi o percentual de erros em relação ao desconhecimento quanto ao fato de que qualquer profissional da rede pública pode fazer a suspeição da doença e realizar os encaminhamentos necessários ao paciente.

De acordo com o Protocolo de Atendimento Hanseníase (Brasil 2007), todos os profissionais que atuam na rede básica de saúde devem estar sempre atentos à suspeição diagnóstica da hanseníase e capacitados para identificar sinais e sintomas em pessoas atendidas na unidade de saúde, na demanda espontânea na comunidade em geral ou em grupos específicos. Essa informação deve ser conhecida pelos voluntários e repassada para os usuários que acessam a REMOB, assim como o conceito que a hanseníase é uma doença que tem cura, já que 14,3\% dos voluntários não tinham essa informação.

Questões relativas ao contato físico, uso de ambientes coletivos e o compartilhamento de objetos pessoais pela pessoa que tem hanseníase ainda causam dúvidas, uma vez que $19,0 \%$ erraram ou desconhecem essa informação. 0 conceito de afastamento social embutido desde os tempos bíblicos, com relatos de que os doentes não podiam participar socialmente das atividades e ou dividir materiais em especial de uso pessoal, faz parte do discurso do sujeito coletivo e é amplamente discutido em estudos qualitativos que bem representam a fala, a dor e a exclusão vividas pelas pessoas que tem ou tiveram hanseníase ${ }^{21,22}$.

Quanto ao item Diagnóstico do questionário, os voluntários souberam responder de forma assertiva, as questões relacionadas à identificação da doença. No entanto o percentual de desconhecimento foi maior em relação a três sinais e sintomas da Hanseníase: "manchas com queda de pêlo", "manchas que pegam pó" e "manchas que coçam". Essas informações são divulgadas, com tiragem limitada, pelos manuais, cartazes, cartilhas, informes do ministério da saúde e órgãos estaduais e municipais, porém são pulverizadas na rede de saúde para profissionais e usuários, podendo por vezes não ser disponibilizado para a população em geral $^{23,24}$. Há de se considerar ainda que essas informações são específicas e que nem todos conhecem as peculiaridades da doença.

Os voluntários tiveram bom desempenho $(85,7 \%)$ nas respostas dos exames realizados no laboratório para verificar a presença de bacilos.

Sobre a Transmissão da hanseníase as questões com maior número de erros ou desconhecimento foram relativas à forma transplacentária, se todas as pessoas que tem contato com o bacilo adoecem e quanto à contagiosidade das formas tuberculóide, dimorfa e virchoviana.

Apesar de ser uma doença milenar a transmissão da hanseníase ainda é assunto 
nebuloso e vem sendo discutido no universo científico. Pesquisadores do mundo inteiro têm investigado a transmissão ${ }^{25-27}$ e aventado outras possibilidades que não só a atualmente aceita de ser a doença transmitida por doentes multibacilares não tratados, como já comprovado por Paul Fine 28 .

Em relação ao Tratamento da hanseníase os voluntários tiveram uma porcentagem de acertos menor em relação aos os outros temas centrais do questionário. 0 desconhecimento foi maior em relação a quantidade de doses mensal para pauci e multibacilares, se o tratamento diminui a eficácia do anticoncepcional, sobre a urina vermelha no dia da dose mensal supervisionada e sobre alteração na cor da pele principalmente em contato com o sol. Sabe-se que o tratamento do paciente com hanseníase é fundamental para curá-lo, fechar a fonte de infecção interrompendo a cadeia da doença, sendo, portanto estratégico no controle da endemia e para eliminar a hanseníase quanto problema de saúde publica $^{29,30}$.

0 tratamento da pessoa com hanseníase, indicado pelo Ministério da Saúde, é a poliquimioterapia padronizada pela Organização Mundial de Saúde, conhecida como PQT, devendo ser realizado nas unidades de saúde ${ }^{29,30}$. Embora a questão do tratamento seja também muito específica, deve ser conhecida pelos voluntários do MORHAN para que saibam repassar e responder as dúvidas para as pessoas que fazem parte de sua rede de ação.

Os resultados desse estudo mostraram que aspectos gerais e específicos da hanseníase ainda são pouco divulgados, e para a ampliação desse conhecimento é necessária maior exposição de seus conceitos por recursos midiáticos como televisão, escolas, cartazes em lugar de grande movimentação de pessoas, palestras, educação continuada e capacitação para os profissionais de saúde e agentes da saúde da família para que a doença seja identificada no início.
Após análise dos resultados ora apresentados, o grupo de pesquisadores propôs uma estratégia de ação que consistia na disponibilização dos materiais educativos, em especial o Álbum Seriado da Fundação Paulista Contra Hanseníase ${ }^{12}$, no site oficial do MORHAN, permitindo que todos os voluntários da REMOB pudessem conhecer de forma ilustrativa e didática os conceitos básicos da hanseníase como proposto pelo instrumento de investigação utilizado nesse estudo. Sugerese que essa ferramenta deva ser disponibilizada de forma permanente no site para que se torne objeto de consulta para o voluntariado e visitantes sempre que se faça necessário.

Em suma, verificou-se que o conhecimento dos voluntários sobre a patologia foi satisfatório. Considera-se importante, estimular o desenvolvimento de uma relação de parceria, de cumplicidade e de transparência de pessoas que se dispõem a atuar no REMOB e que há necessidade de educação permanente no voluntariado, sendo prioritário investir em capacitação e educação continuada dessas pessoas.

A proposta é trazer à tona e promover a discussão das várias facetas da hanseníase de forma a contribuir para a formação dos voluntários do MORHAN que atuam especialmente na política do controle da endemia, e luta pelos direitos das pessoas diagnosticadas com a doença. Diante dos resultados aqui apresentados, ações que estimulem a transdisciplinariedade $\mathrm{e}$ incentivem a contribuição de parceiros nãotécnicos podem ser efetivadas com o propósito de garantir direitos e melhorar a qualidade do atendimento ao doente e suas famílias.

Como limitação desse estudo aponta-se que no momento de aplicação do pré-teste, nem todos os voluntários do MORHAN estavam cadastrados no REMOB, impossibilitando assim a participação de todos os voluntários dessa complexa rede que luta pela eliminação da hanseníase no país e no exterior. 


\section{CONCLUSÃO}

0 conhecimento sobre hanseníase foi maior nas questões referentes à Diagnóstico da doença e menor nos itens sobre Tratamento.

As afirmativas que tiveram maior erro ou desconhecimento, embora sejam bastante específicas e técnicas, devem ser alvo de investimento para educação permanente.

Sugere-se como uma das estratégias de ação a disponibilização de materiais educativos no site oficial do MORHAN para melhor atuação no voluntariado.

\section{REFERÊNCIAS}

1. World Health Organization. Leprosy: global situation [Internet]. Geneva: WHO; 2012 [citado em 2014 Jan 30]. Disponível em: http://www.who.int/lep/situation/en/.

2. Organização Mundial da Saúde. Estratégia global aprimorada para redução adicional da carga da hanseníase: período do plano 2011-2015. Brasília: OMS; 2010.

3. Ministério da Saúde (Br), Secretaria de Vigilância em Saúde. Departamento de Vigilância em Doenças Transmissíveis. Plano integrado de ações estratégicas de eliminação da hanseníase, filariose, esquistossomose e oncocercose como problema de saúde pública, tracoma como causa de cegueira e controle das geohelmintíases: plano de ação 20112015. Brasília: Ministério da Saúde; 2012.

4. Eidt LM. Breve história da hanseníase: sua expansão do mundo para as Américas, o Brasil e o Rio Grande do Sul e sua estratégia na saúde pública brasileira. Saúde Soc. 2004; 13(2):76-88.

5. Cordeiro TL, Frade MAC. Leprosy: education as first priority. J Neurosci Rural Pract. 2014; 5(supl 1):S3-S4.

6. Rocha CMV. Educação em saúde: breve histórico e perspectivas. In: Ministério da Saúde (Br). Coletânea de saúde e educação em saúde. Brasília: Ministério da Saúde; 1989.

7. Ministério da Saúde (Br). Secretaria de Políticas de Saúde. Departamento de Gestão de Políticas Estratégicas. Área Técnica de Dermatologia Sanitária. Diretrizes nacionais para elaboração de programas de capacitação para equipe de saúde da rede básica atuar nas ações de controle da hanseníase pela área técnica de dermatologia sanitária. Brasília: ATDS; 2000.

8. Melo MC, Souza AL, Leandro EL, Mauricio HÁ, Silva ID, Oliveira JMO. A educação em saúde como agente promotor de qualidade de vida para o idoso. Ciênc Saúde Coletiva [Internet]. 2009 [citado em 22 jan 2015]; 14(supl 1):1579-86. Disponível em:

http://www.scielo.br/scielo.php?script=sci_arttext \&pid=S141381232009000800031\&lng=en\&nrm=i so.

9. Morhan: Movimento de Reintegração das Pessoas Atingidas pela Hanseníase [Internet]. Rio de Janeiro: Morhan; 2011 [citado em 21 jan 2015]. Disponível em: http://www.morhan.org.br/institucional.

10. FormFacil: Pré-teste [Internet]. 2001 [citado em 21 jan 2015]. Disponível em: http://www.formfacil.com/susitn/preteste.

11. Marciano LHSC, Prado RBL, Quaggio CMP, Nardi SMT. Proposta pedagógica para aprimorar os conceitos básicos em hanseníase: álbum seriado como um recurso no processo de orientação. Hansen Int. 2008; 33(2):17-24.

12. Fundação Paulista Contra Hanseníase. Álbum seriado: 2004. São Paulo: Fundação Paulista Contra Hanseníase; 2004.

13. Xavier RCM, Costa RO. Relações mútuas entre informação e conhecimento: o mesmo conceito? $\mathrm{Ci}$ Inf. 2010; 39(2):75-83.

14. Cabello KSA, Rocque LL, Sousa ICF. Uma história em quadrinhos para o ensino e divulgação da hanseníase. Rev Electrón Ensen Ciências. 2010; 9(1):225-41.

15. Barros DMV, Neves C, Seabra CNF, Moreira JA, Henriques S. Educação e tecnologias: reflexão, inovação e práticas [Internet]. Lisboa: Edição dos Autores; 2011 [citado em 15 Fev 2015]. Disponível em:

http://repositorioaberto.uab.pt/handle/10400.2/2 771.

16. Souza MGG, Santo FHE. O Olhar que olha o outro: um estudo com familiares de pessoas em quimioterapia antineoplásica. Rev Bras Cancerol. 2008; 54(1):31-41.

17. Opromola DVA. Noções de hansenologia. Bauru: Centro de Estudos Dr. Reynaldo Quagliato; 2000.

18. Ferreira JS, Neumann AS, Ferreira ABR, Rangel $\mathrm{CP}$, Uzedo CCD, Fonseca AH, et al. Análise da persistência do mycobacterium leprae em amblyomma cajennense e rhodnius prolixus após infecção por alimentação artificial. Hansen Int. 2012; 37(2):18.

19. Deps PD, Antunes JMAP, Faria C, Bührer-Sékula S, Camargo ZP, Opromola DV, et al. Pesquisa de anticorpos anti PGL-I através de ELISA em tatus 
selvagens do Brasil. Rev Soc Bras Med Trop. 2008; 41(supl2):73-6.

20. Trabulsi LR, Althertum F. Microbiologia. 5ed. Rio de Janeiro: Atheneu; 2008.

21. Nunes JM, Oliveira EM, Vieira NFC. Ter hanseniase: percepções de pessoas em tratamento. Rev RENE. 2008; 9(4):99-106.

22. Rocha ACRP, Landim FLP, Caprara A, Lefèvre A, Lefèvre $F$. The collective discourse of a former hansen's disease patient living in an old colony in northeastern Brazil. Interface Comun Saúde Educ. 2011; 15(36):213-23.

23. Santos AK, Monteiro S, Rozemberg B. Significados e usos de materiais educativos sobre hanseníase segundo profissionais de saúde pública do município do Rio de Janeiro, Brasil. Cad Saúde Pública. 2009; 25(4):857-67.

24. Ministério da Saúde (Br). Fundação Oswaldo Cruz. Banco de materiais educativos sobre hanseníase [Internet]. Rio de Janeiro: FIOCRUZ; 2010 [citado em 12 fev 2015]. Disponível em: http://hanseniase.icict.fiocruz.br/index.php.

25. Prevedello FC, Mira MT. Hanseníase: uma doença genética? An Bras Dermatol. 2007; 82(5):451-9.

26. Jarduli LR, Alves HV, Souza-Santana FC, Marcos EVC, Pereira AC, Dias-Baptista IMF, et al. Influence of KIR genes and their HLA ligands in the pathogenesis of leprosy in a hyperendemic population of Rondonópolis, Southern Brazil. BMC Infect Dis. 2014; 14(1):438.

27. Grant AV, Cobat A, Van Thuc N, Orlova M, Huong NT, Gaschignard J, et al. CUBN and NEBL common variants in the chromosome $10 \mathrm{p} 13$ linkage region are associated with multibacillary leprosy in Vietnam. Hum Genet. 2014; 133(7):883-93.
28. Fine PEM, Stern JA, Ponnighaus JM, Bliss L, Saui J, Chihana A, et al. Household and dwelling contact as risk factors for leprosy in the Northern Malawi. Am J Epidemiol. 1997; 146(1):91-102.

29. Souza, LR. Condicionantes sociais na delimitação de espaços endêmicos de hanseníase. Tese [Doutorado]. São Paulo: Universidade de São Paulo; 2012.

30. Ministério da Saúde (Br). Portaria n. 198, 15 de outubro de 2010. Aprova as diretrizes para vigilância, atenção e controle da hanseníase. Seção 1. Ministério da Saúde (Brasil). Portaria no 3.125, de 7 de outubro de 2010. Aprova as Diretrizes para Vigilância, Atenção e Controle da Hanseníase [Internet]. Brasília: Ministério da Saúde; 2010 [citado em 21 jan 2015]. Disponível em: http://bvsms.saude.gov.br/bvs/saudelegis/gm/20 10/prt3125_07_10_2010.html.

CONTRIBUIÇÕES
Susilene Maria Tonelli Nardi realizou a
coordenação, concepção, planejamento, análise e
interpretação dos dados, redação final do artigo.
Heloisa da Silveira Paro Pedro participou no
desenho do estudo, análise e interpretação dos
dados, redação final do artigo. Luisa Arantes
Loureiro atuou no desenho do estudo, elaboração
do instrumento de coleta, coleta de dados e
redação final do artigo. Lucia Helena Soares
Camargo Marciano desenvolveu o desenho e
concepção do estudo, planejamento de execução,
revisão crítica e redação final do artigo. Artur
Custódio Correa de Sousa participou na
concepção e desenho do estudo, elaboração do
instrumento de coleta, coleta de dados e redação
final do artigo. Vânia Del'Arco Paschoal atuou no
desenho e concepção do estudo, planejamento de
execução, revisão crítica e redação final do artigo.

\footnotetext{
Como citar este artigo (Vancouver):

Nardi SMT, Pedro HSP, Loureiro LA, Marciano LHSC, Sousa ACC, Paschoal VD. Rede virtual: ferramenta para analisar o conhecimento dos voluntários do MORHAN sobre hanseníase. REFACS [Online]. 2016 [citado em (inserir dia, mês e ano de acesso)]; 4(2). Disponível em: (link de acesso). DOI: 10.18554/refacs.v4i2.1068.

Como citar este artigo (ABNT):

NARDI, S. M. T. et al. Rede virtual: ferramenta para analisar o conhecimento dos voluntários do MORHAN sobre hanseníase. REFACS, Uberaba, MG, v. 4, n. 2, p. 162-171, 2016. Disponível em: (link de acesso). DOI: 10.18554/refacs.v4i2.1068. Acesso em: (inserir dia, mês e ano de acesso).

Como citar este artigo (APA):

Nardi, S. M. T., Pedro, H. S. P., Loureiro, L. A., Marciano, L. H. S. C., Sousa, A. C. C. \& Paschoal, V.D. (2016). Rede virtual: ferramenta para analisar o conhecimento dos voluntários do MORHAN sobre hanseníase. REFACS, 4(2), 162-171. Recuperado em (dia), (mês), (ano) de (link de acesso). DOI: 10.18554/refacs.v4i2.1068.
} 